\title{
An Algebraic Approach to DC Railway Electrification Verification
}

\author{
Eugenio Roanes-Lozano • Rubén \\ González-Martín · Javier Montero
}

Received: date / Accepted: date

\begin{abstract}
Gröbner bases have been applied to a number of problems related to the verification of Knowledge-Based Systems (KBS) and other problems within graph theory. In particular, the authors have developed in previous papers algebraic approaches to decide whether a situation in a railway interlocking system is safe or not. These algebraic approaches stand out because of the briefness of the code (as they use implementations of well-known algorithms for solving linear or algebraic systems provided by computer algebra systems). The authors have also developed a matrix-based computer tool that helps an expert to check whether a proposed railway electrification scenario (given through the topology of the railway station and the position of the isolation devices and the position and state of the "electrical bypasses" and feeders) fulfills the mandatory requirements of the Spanish railway infrastructure administrator (ADIF) for 3,000 V railway electrifications or not. The second author works in the field of railway electrifications and he compares the present-day methods for verification of railway electrifications with the way KBS were verified in the past (manually by experts). In this article we approach this latter problem using algebraic techniques. The new computer tool is based on an algebraic translation of the problem (instead of based on the use of matrices)
\end{abstract}

Eugenio Roanes-Lozano

Instituto de Matemática Interdisciplinar \& Depto. de Didáctica de Ciencias Experimentales, Sociales y Matemáticas, Facultad de Educación, Universidad Complutense de Madrid c/ Rector Royo Villanova s/n, E-28040 Madrid, Spain

ORCID ID: 0000-0002-0880-6610

Tel.: +34-91-3946248

Fax: +34-91-3946157

E-mail: eroanes@mat.ucm.es

Rubén González-Martín

Ineco (\& Universidad Politécnica de Madrid)

Avda. Partenón, 4-6 $1^{a}$ Planta Puerta 3, E-28042, Madrid, Spain

ORCID ID: 0000-0002-9789-5423

Javier Montero

Depto. de Estadística e Investigación Operativa, Facultad de Ciencias Matemáticas,

Universidad Complutense de Madrid

Plaza de Ciencias s/n, E-28040 Madrid, Spain

ORCID ID: 0000-0001-8333-2155 
and is really simple and fast. Determining which electrification sections are under electric tension is computed solving linear systems (because in this case the graph is undirected and no polynomials of degree greater than 1 arise in the algebraic translation, so it is not necessary to use Gröbner bases, unlike in the two problems mentioned in the beginning of the Abstract). Therefore far bigger railway facilities can be addressed than if non-linear systems were involved.

Keywords Railway electrifications · graphs · verification · polynomials · computer algebra systems

Mathematics Subject Classification (2010) 90C35 -34A30 - 68T35

\section{Introduction}

Railways are guided transportation systems [10,14] and, consequently, problems such as railway traffic management are far simpler than in road transportation.

The second author works in the field of railway electrifications and compares the present-day methods for verification of railway electrifications with the way Knowledge-Based Systems (KBS) were verified in the past (manually by experts). Therefore we developed a matrix approach to DC railway electrification verification that was presented at the International Conference on Computational Science ICCS'2017 [19]. The approach detailed in this new article is algebraic and was presented at the 6th European Seminar on Computing ESCO'2018 [20].

This new algebraic approach is based on the use of polynomials and is similar to those used by the authors for:

- KBS verification [21] (whose underlying logic is either Boolean or modal multivalued $[1,6,8,13])$,

- decision making in a railway interlocking system using Gröbner bases [22].

Nevertheless, electrical connectivity, unlike knowledge extraction in KBS or the possible movements of trains in a railway layout, does not take "sense" into account (in a railway layout a semaphore can forbid a movement to trains, e.g. from section $A$ to section $B$, meanwhile the movement in the opposite "sense", e.g. from section $B$ to section $A$, can be allowed). This makes the implementation considered in this new article far simpler than those used in [21] for KBS verification and in [22] for decision making in a railway interlocking systems, since the polynomial system that translates the problem is now linear (as we'll detail afterwards). Therefore using Gröbner bases [4] is not required, and the processes are much faster. The new approach has also been implemented in the computer algebra system Maple $[5,9,12,15,23]$, but could be ported to other systems [24].

\section{Some notes about railway electrifications}

2.1 About the different electrification systems

Unlike what happens in North America, main lines in Europe are nowadays electrified. Although there are different electrification systems (using a side rail or 
central rail as the other pole, using three phase alternating current $(\mathrm{AC})^{1}$, etc.), almost all main lines use the rails as ground pole and the other pole is the overhead contact system (also denoted overhead line or catenary). It is denoted this latter way because of the shape of the messenger wire, that supports the contact wire, that must be completely horizontal (Figure 1). Note that in some low speed networks (like subways and tramway networks) sometimes an overhead line with only one wire is used. Let us finally mention that electric trains collect the electric current from the catenary using a device denoted pantograph.

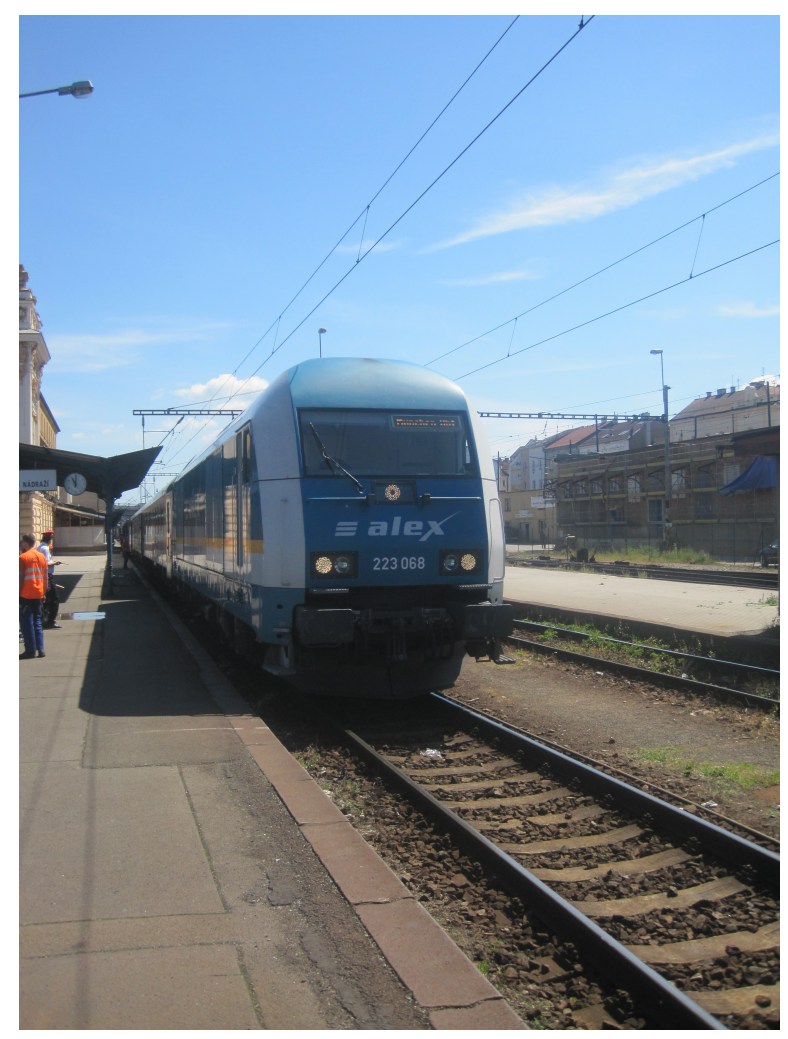

Fig. 1 A diesel locomotive at Pilsen station. The messenger and contact wires of the catenaries above the train and above the track to the right are clearly distinguishable.

Three are the electrification systems most commonly use for railway main lines (tramways and subways usually adopt lower voltages) $[16,18]$ :

1 Three-phase AC electrifications were experimented by the military in Germany in the beginning of the XXth century (they set a railway speed world record) and some lines existed in South East Spain and North Italy that survived till the second half of the XXth century. Using three-phase electrifications has many advantages from the electrical point of view, but has great disadvantages from the practical point of view: either two overhead lines or three side lines have to be used 
- $1.5 K V$ Direct Current (DC) or $3 K V$ DC. Used in Spain, France, Italy, Belgium, Czech Republic, etc.

- $15 K \mathrm{~K}$ AC, $16 \frac{2}{3} \mathrm{~Hz}$. Used in Central and Northern Europe: Germany, Austria, Norway, Sweden and Switzerland.

- $25 \mathrm{KV} \mathrm{AC}, 50 \mathrm{~Hz}$. Used in France, Portugal and the new high speed lines of other countries such as Spain, Belgium and Italy, etc.

(the examples of use given above are not exhaustive and refer only to European countries).

Low voltage DC has a clear disadvantage since it requires rectification and a strong reduction from the voltage of the industrial electrical network (usually $220 K V$ or $400 K V)$. Moreover, the contact wire needs to have a bigger section and transmission losses are much higher. Consequently, modern railway electrifications normally use high voltage AC (unless it is an extension of an existing railway network that already uses DC).

2.2 Some notes about isolation devices and "electrical bypasses" at railway electrifications

Just in case there is any problem with the overhead contact system, it is splitted into isolated parts, denoted electrification sections. A special type of electrification section is the neutral section.

Different kinds of isolation devices can be installed between electrification sections:

- Section insulator (a.k.a. insulator):

- Air-gap insulator (a.k.a. insulated overlap): $\longrightarrow$.

and there are different kinds of "electrical bypasses" of the isolation devices:

- Earthing disconnector (a.k.a. grounded circuit breaker): $\bullet$

- Load disconnector (a.k.a. ungrounded circuit breaker): $\bigotimes$.

- Remote disconnector (a.k.a. telecommanded not-grounded circuit breaker): (Q).

We should also mention that some electrification sections receive electricity from the electrical substation through a feeder. Finally, at an end buffer there is always an stop to electric traction.

2.3 Summary of the mandatory requirements of ADIF (Spanish railway infrastructure administrator) for $3 K V D C$ electrifications

The following list of requirements is the same as the one considered in [19]:

i) Neutral zones must be installed at the end or ends of the stations (on both sides in case it is a through station and on one side in case it is a terminus station).

ii) Section insulators have to be installed in all the connections between main tracks.

iii) There has to be an earthing disconnector at tracks with a buffer stop where maintenance labours could take place. 
iv) There must be a section insulator in all turnouts that connect main tracks with the rest of tracks of the station.

v) That all the electrified tracks of the station can be fed is compulsory.

vi) That all sidings beside a main track can be fed through a load disconnector from that main track is required.

vii) It is allowed to install air-gap insulators and disconnectors in the main tracks of long stations in order to split them into shorter electrification sections.

viii) Even (respectively odd) sidings have to be fed from the even (respectively odd) main track or even (respectively odd) sidings.

\section{Modeling the electrical connectivity of the railway facility}

3.1 Description of the model and main assert

The description of the electrical situation of the railway facility at a certain instant can be splitted into two categories: there are fixed issues and issues that change along time. The fixed issues are:

- the topology of the electrification of the station,

- the position of the isolation devices, "electrical bypasses" and feeding devices, and the issues that change along time are:

- whether the "electrical bypasses" allow electric current to pass through them or not,

- and whether the feeders feed the corresponding electrification section or not.

Therefore, the main assert regarding the connectivity between two electrification sections is the following:

electricity will pass from an electrification section, $a$, to another electrification section, $b \Longleftrightarrow \alpha \wedge(\beta \vee \gamma)$

where:

- $\alpha \equiv$ it is topologically possible to pass from electrification section $a$ to electrification section $b$

- $\beta \equiv$ there is no isolation device between electrification section $a$ and electrification section $b$

- $\gamma \equiv$ there are isolation devices between electrification section $a$ and electrification section $b$ but they are all bypassed by "electrical bypasses" devices allowing electricity passing by (along, at least, one path).

3.2 The algebraic approach and the algorithm

The proposed approach is independent form the topology of the station has no upper boundary for the number of electrification sections.

It uses undirected graphs that are translated into algebra as follows:

- One (or both polynomials) $a-b, b-a$ represent that electrification sections $a$ and $b$ are connected (from the electrical point of view). 
- We shall consider the set of such polynomials for the whole layout under study (it is a set of linear polynomials, that is, a system of linear equations).

- Note that these approach automatically considers the reflexive-transitive closure of the undirected graph.

- To assert that an electrification section, $a$, receives electricity (from the feeder or from an adjacent electrification section in the main line) is represented by the assigning $a:=1$.

- The electrification sections that have the value 1 when solving the system of linear equations are those under electric tension.

And the algorithm proposed is the following:

1) Consider the set of polynomials $(a-b$ and/or $b-a)$ that gives the topology of the electrification undirected graph.

2) Eliminate from that set those polynomials representing the positioning of the the isolation devices (section insulators and air-gap insulators).

3) Add to that set the polynomials representing the positioning of the electrical bypasses (earthing disconnectors, load disconnectors and remote load disconnectors) that are in the "ON" situation.

4) Finally, add the assignings of the form $a:=1$ (electrification sections that are under electric tension because they are connected to a feeder or because they are connected to an electrification section of the main line that is under electric tension).

5) Solve the system. The variables that are valued as 1 give us the electrification sections that are under electric tension (the other variables will be unassigned).

\section{Implementation}

Apart form the data introduction procedures, the package provides three auxiliary procedures for checking the correctness of the electrification situation easier:

- Return the secondary track electrification sections from the (given) list of main tracks.

- Turn all existing ON/OFF circuit breakers OFF, except those between electrification sections of the same main track, that are ALL turned ON

- Turn all existing ON/OFF circuit breakers OFF, except those between electrification sections of the given list.

- Return the names of electrification sections under electric tension (according to the fed ones introduced).

- Return the powered / unpowered electrification sections of main / secondary tracks.

\section{Examples}

Let us consider, for example, the electrification of a railway station represented in Figure 2 (the symbols were introduced in Section 2.2). Different scenarios are analyzed below in self explanatory examples (although in Maple code, they are easy to follow, just take into account that $>$ is the input prompt and \# is used to include comments). Sometimes dots are included for the sake of brevity. 


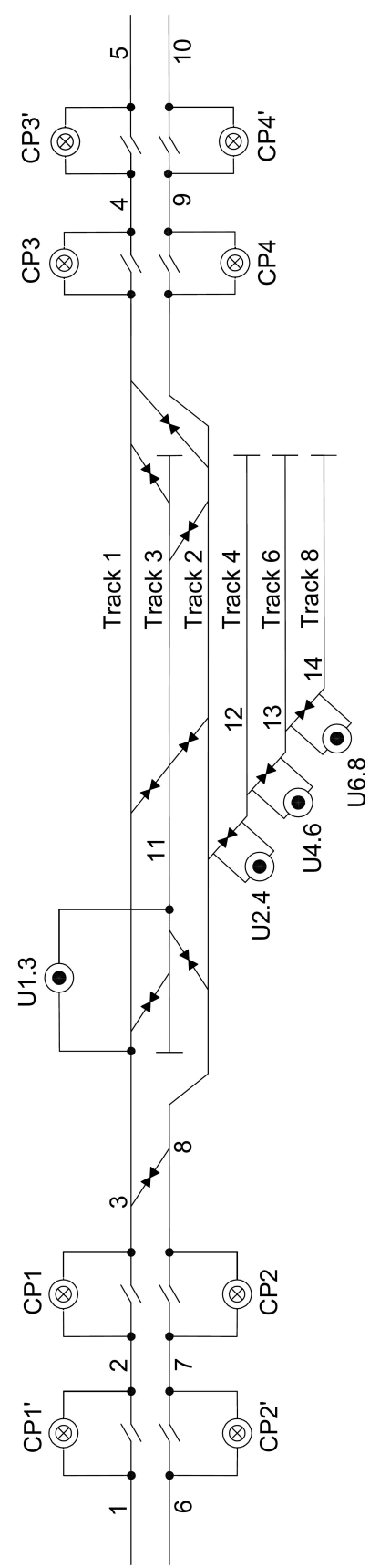

Fig. 2 An example of a station. The numbers preceded by "Track" are the numbers of the tracks. The isolated numbers denote the number we have assigned to the electrification sections. 
Example 1 Let us load the code and introduce the fixed data using initialize, which input is the list of names of the electrification sections in main track 1, the list of names of electrification sections on main track 2, and the number of electrification sections on secondary tracks. Afterwards a first scenario (with all "electrical bypasses" ON) is declared.

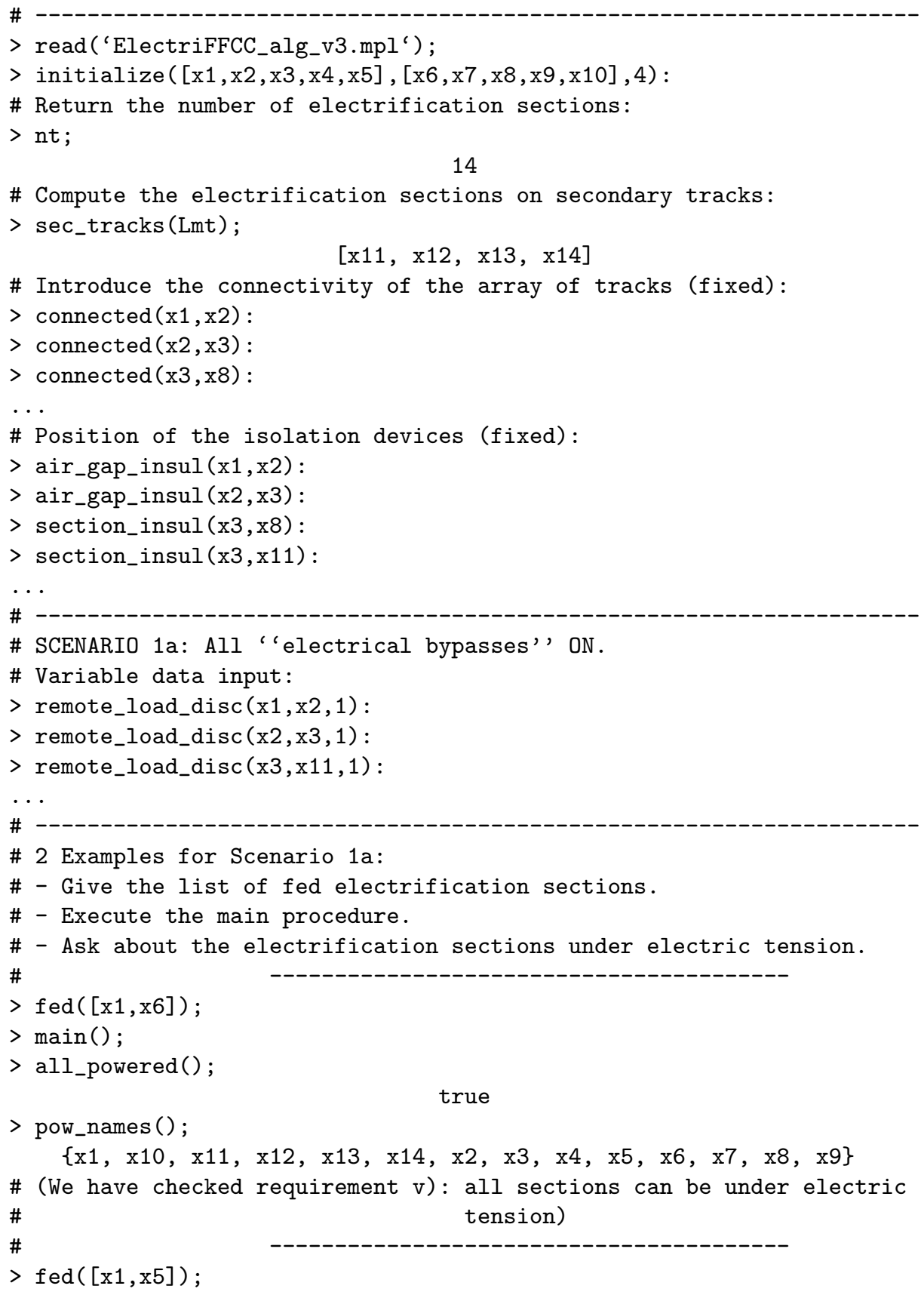


$>\operatorname{main}()$;

$>$ all_powered () ;

false

$>$ pow_names ();

$\{\mathrm{x} 1, \mathrm{x} 11, \mathrm{x} 2, \mathrm{x} 3, \mathrm{x} 4, \mathrm{x} 5\}$

\# (We have checked requirement vii) for the odd siding $x 11$ : it can

\# receive electricity from the odd main track)

Example 2 Let us turn OFF three "electrical bypasses".

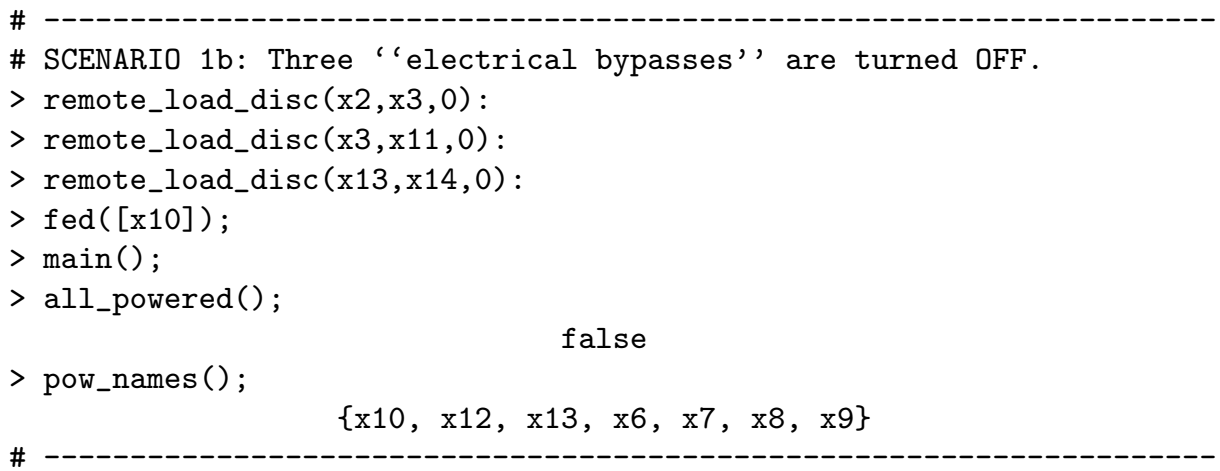

Example 3 Of all the existing ON/OFF circuit breakers, only the circuit breakers between electrification sections of the main track are turned ON, the rest are OFF (this is directly performed by only_main_tracks().

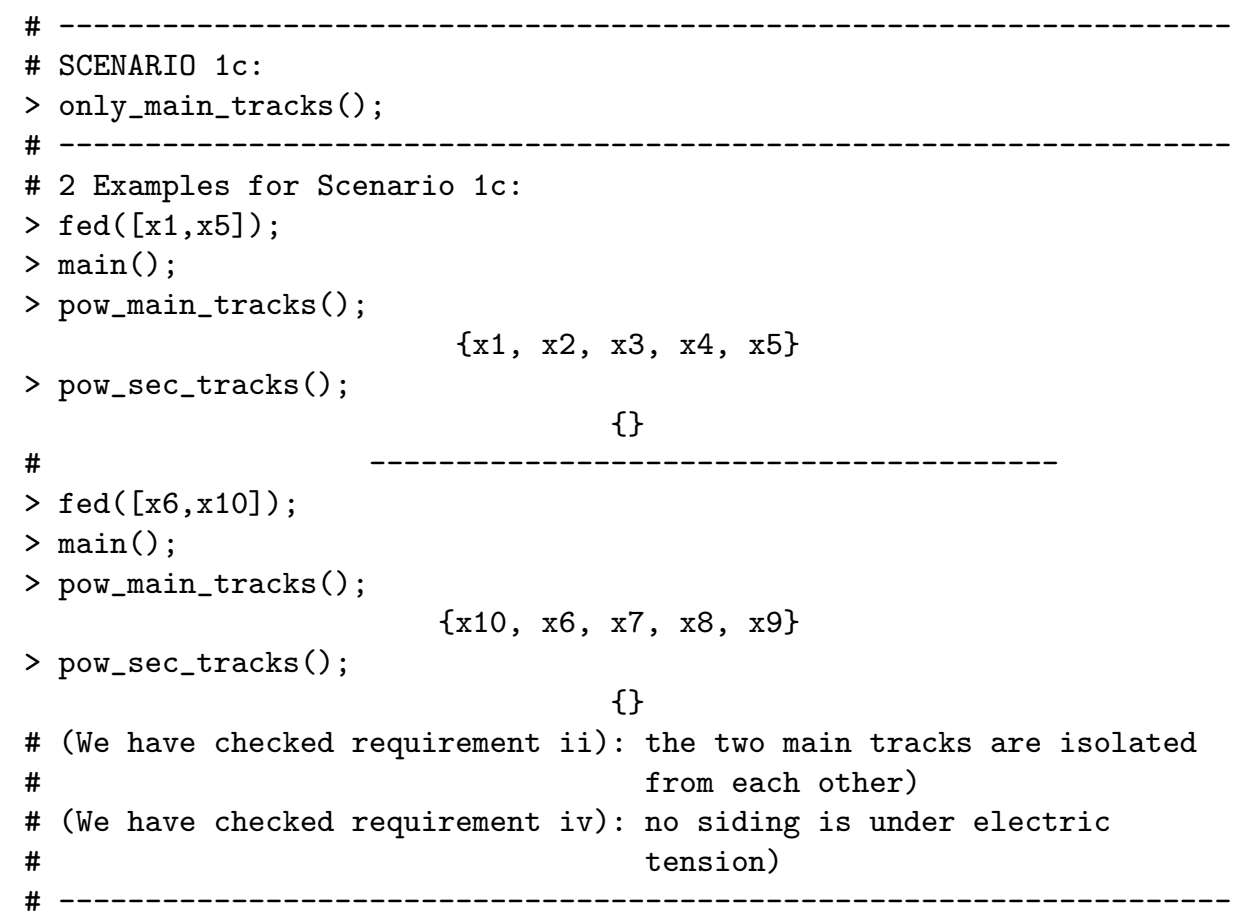




\section{Conclusions and Future Work}

The work presented here is able to perform computations that can help in the the verification process of railway electrifications. Let us note that errata are often found in manual approximations to verification. A curious example is [3], where an unacceptable error was found when verifying the railway interlocking system of a certain simple railway facility.

There are very many works related to railway electrifications from different points of view. For instance, the authors of [2] compare different railway electrification systems and underlines the advantages of low frequency AC ones. Meanwhile in [17] the advantages of using DC in electrified metropolitan railways is studied. There are several works on energy optimization, for instance using genetic algorithms [7] or the advantages of electrical smart grids [11]. But we have found no publication addressing the verification of a railway electrification (apart from the aforementioned [19]). Moreover, our work focuses on ADIF specific mandatory requirements (although it could be adapted to other infrastructure administrations). We have tested the implementation on small real examples, such as that shown in Section 5, and we plan to test the implementation extensively.

A future work is to completely automatize the testings of the expert. Another possibility is to address the problem of $A C$ ADIF electrifications (that have different requirements).

Acknowledgements This work was partially supported by the research projects TIN201566471-P (Government of Spain) and CASI-CAM S2013/ICE-2845 (Comunidad Autónoma de Madrid).

We would also like to thank Ineco, the company where the second author works, for facilitating the development of this work.

\section{References}

1. Alonso-Jiménez, J., Aranda-Corral, G., Borrego-Díaz, J., Fernández-Lebrón, M., HidalgoDoblado, M.: A logic-algebraic tool for reasoning with knowledge-based systems. Journal of Logical and Algebraic Methods in Programming

2. Bhargava, B.: Railway electrification systems and configurations. In: 1999 IEEE Power Engineering Society Summer Meeting. Conference Proceedings (Cat. No.99CH36364), vol. 1, pp. 445-450 vol.1 (1999). DOI 10.1109/PESS.1999.784389

3. Boralv, A.: Case study: formal verification of a computerized railway interlocking. Formal Aspects of Computing 10, 338-360 (1998). DOI 10.1007/s001650050021

4. Buchberger, B.: Bruno Buchberger's PhD thesis 1965: An algorithm for finding the basis elementals of the residue class ring of a zero dimensional polynomial ideal. Journal of Symbolic Computation 41(3-4), 475-511 (2006). DOI 10.1016/j.jsc.2005.09.007

5. Buckhardt, W.: First Steps in Maple. Springer-Verlag, New York - Berlin - Heidelberg (1994)

6. Burke, R.: Knowledge-based recommender systems. In: Encyclopedia of Library and Information Science, vol. 69/32, pp. 180-200 (2000)

7. Chang, C.S., Wang, W., Liew, A.C., Wen, F.S., Srinivasan, D.: Genetic algorithm based bicriterion optimisation for traction substations in DC railway system. In: Proceedings of 1995 IEEE International Conference on Evolutionary Computation, vol. 1, pp. 11-16 (1995). DOI 10.1109/ICEC.1995.489111

8. Chazarain, J., Riscos, A., J.A., A., Briales, E.: Multi-valued logic and Gröbner bases with applications to modal logic. Journal of Symbolic Computation 11, 181-194 (1991). DOI 10.1016/S0747-7171(08)80043-0

9. Corless, H.: Essential Maple. An Introduction for Scientific Programmers. Springer-Verlag, New York (1995) 
10. de Fontgalland, B.: The World Railway System. Cambridge University Press, Cambridge, UK (1984)

11. Pilo de la Fuente, E., Mazumder, S.K., González-Franco, I.: Railway electrical smart grids: An introduction to next-generation railway power systems and their operation. IEEE Electrification Magazine 2(3), 49-55 (2014). DOI 10.1109/MELE.2014.2338411

12. Heck, A.: Introduction to Maple. Springer-Verlag, New York - Berlin - Heidelberg (2003)

13. Laita, L., de Ledesma, L.: Knowledge-based systems verification. In: Encyclopedia of Computer Science and Technology, pp. 253-280 (1997)

14. Losada, M.: Curso de ferrocarriles. E.T.S. Ingenieros de Caminos, Canales y Puertos, Madrid (1987)

15. Maplesoft: Maple User Manual. Maplesoft, Waterloo Maple Inc., Waterloo, Canada (2018)

16. Olaizola-Elordi, J.: La electrificación de los ferrocarriles de vía estrecha. Vía Libre 616, 22-25 (2017)

17. Pecharromán, R.R., López-López, A., Cucala, A.P., Fernández-Cardador, A.: Riding the rails to DC power efficiency. IEEE Electrification Magazine 2(3), 32-38 (2014). DOI 10.1109/MELE.2014.2333684

18. R., A.: Electrificación: Catenaria en la red española. Vía Libre 616, 15-21 (2017)

19. Roanes-Lozano, E., González-Martín, R.: Matrix approach to DC railway electrification verification. Procedia Computer Science 108, 1424-1433 (2017). DOI 10.1016/j.procs. 2017.05.226

20. Roanes-Lozano, E., González-Martín, R., Montero, J.: A knowledge-based system for DC railway electrification verification. In: Book of Abstracts ESCO2018 6th. European Seminar on Computing, p. 140 (2018)

21. Roanes-Lozano, E., Laita, L., Hernando, A., Roanes-Macías, E.: An algebraic approach to rule based expert systems. Rev. R. Acad. Cien. Serie A. Mat. (RACSAM) 104/1, 19-40 (2011). DOI 10.5052/RACSAM.2010.04

22. Roanes-Lozano, E., Roanes-Macías, E., Laita, L.M.: Railway interlocking systems and Gröbner bases. Mathematics and Computers in Simulation 51(5), 473-481 (2000). DOI 10.1016/S0378-4754(99)00137-8

23. Roanes-Macías, E., Roanes-Lozano, E.: Cálculos Matemáticos con Maple V.5. Ed. Rubiños, Madrid (1999)

24. Wester, M., (Ed.): Computer Algebra Systems: A practical Guide. John wiley \& Sons, Chichester, UK (1999) 